\title{
CONVENTIONAL TECHNIQUE V/S TWO-STAGE TECHNIQUE FOR EXTRACTION OF IMPACTED LOWER THIRD MOLAR- A COMPREHENSIVE COMPARISON
}

\author{
Tripthi Prakash Shetty1, Prakyath Malli² \\ ${ }^{1}$ Senior Lecturer, Department of Oral and Maxillofacial Surgery, A. B. Shetty Memorial Institute of Dental Sciences, Nitte University, \\ Mangalore. \\ ${ }^{2}$ Senior Lecturer, Department of Prosthodontics, Yenepoya Dental College, Yenepoya University, Mangalore.
} \begin{abstract}
BACKGROUND

Extraction of impacted mandibular third molars is a routinely performed procedure and is commonly associated with the worrisome complications of inferior alveolar nerve (IAN) injury. Many different techniques have been advocated over the conventional extraction procedures to overcome these complications. The two-stage technique for removal of impacted mandibular molars is one such novel technique that has been discussed in literature, but not very often used. Our study aims to compare this technique with the conventional technique and evaluate its advantages if any.

The aim of this prospective comparative study was to evaluate the pros and cons of the two-stage technique of extraction of impacted lower third molar to the conventional technique in terms of Inferior alveolar nerve (IAN) injury, healing post-operatively and patient's compliance as a whole.
\end{abstract}

\section{MATERIALS AND METHODS}

This clinical prospective non-randomised controlled trial involved twenty patients diagnosed with horizontally impacted lower third molar in close proximity of the inferior alveolar nerve placed. The sample size was chosen as per convenience. The subjects were placed arbitrarily into two groups of ten patients- Test group and Control group respectively. The control group underwent the conventional technique of transalveolar extraction for the impacted lower molar and the test group underwent the two-stage technique for removal of the same.

\section{RESULTS}

Between June 2014 - September 2016, twenty patients were included in the study; 8 of the patients were women (40\%) and 12 men $(60 \%)$ with a mean age of 25 years. The test and control groups showed no significant difference in the incidence of IAN injury $(10 \% \mathrm{v} / \mathrm{s} 20 \%$ respectively). Healing of the soft tissues was better in the control group, as was the patient's compliance and operator satisfaction.

\section{CONCLUSION}

Both the techniques studied have their own advantages and disadvantages with neither techniques being significantly better than the other. Judicious use of the two-stage technique, two-stage method for extracting a horizontally impacted lower third molar however can be useful for decreasing the rate of post-operative complication of inferior alveolar nerve injury.

\section{KEYWORDS}

Two-Stage Technique of Extraction, IAN Injury, Conventional Technique, Third Molar Extraction.

HOW TO CITE THIS ARTICLE: Shetty TP, Malli P. Conventional technique v/s two-stage technique for extraction of impacted lower third molar- a comprehensive comparison. J. Evolution Med. Dent. Sci. 2017;6(91):6521-6527, D0I: $10.14260 /$ jemds/2017/1415

\section{BACKGROUND}

Extraction of impacted mandibular third molars is the mainstay procedure of Oral and Maxillofacial surgery. As simple, humble and routine as the procedure seems, every case poses its own challenge more so pertaining to horizontally impacted lower third molars. The surgical procedure of removal of these teeth is associated with various complications such as postoperative infection, ${ }^{1}$ alveolar fracture $^{2}$ and dysfunction of lingual and/ or inferior alveolar nerves. $^{3}$ The rate of overall complications has been reported to range from $2.6 \%$ to $30.9 \% \%^{4-7}$ and lingual and/ or inferior

'Financial or Other Competing Interest': None.

Submission 27-09-2017, Peer Review 12-11-2017,

Acceptance 18-11-2017, Published 27-11-2017.

Corresponding Author:

Dr. Tripthi Prakash Shetty,

Senior Lecturer,

Department of Oral and Maxillofacial Surgery,

A. B. Shetty Memorial Institute of Dental Sciences,

Nitte University, Derlakatte, Mangalore 575018.

E-mail: tripthi12@gmail.com

DOI: $10.14260 /$ jemds $/ 2017 / 1415$ alveolar nerve injury occurs with a frequency of $0.4 \%$ to $5.5 \%{ }^{7,8}$ Damage to the inferior alveolar nerve (IAN) during third molar extraction is a major concern for patients and clinicians. A wide range of the incidence of temporary and permanent neurologic disturbances of the IAN as a consequence of mandibular impacted third molar extraction has been reported in the literature. The incidence of inferior nerve injury is associated with the anatomical relationship between the root(s) of the third molar and the inferior alveolar nerve canal, and the rate of such nerve injury dramatically increases when the $\operatorname{root}(\mathrm{s})$ is close to the canal.9,10 Therefore, modification of the surgical technique for lower-third molar extraction is required to decrease the incidence of nerve injury, especially when the third molar is horizontally impacted and the root is close to the inferior alveolar nerve canal.

To reduce this risk, several approaches have been proposed. Some authors advocated orthodontic-assisted extraction of the impacted mandibular third molars..$^{7-9}$ Others introduced partial odontectomy, that is the surgical removal of the anatomic crown leaving the roots in place. ${ }^{10-12}$ This study is to compare the conventional transalveolar technique of 
removal of horizontally impacted lower third molars in close proximity to IAN nerve to a novel surgical procedure of a twostage approach for the same.

\section{MATERIALS AND METHODS}

The study was a clinical non-randomised prospective controlled trial involving twenty patients visiting the Department of Oral and Maxillofacial Surgery at ABSMI Dental Sciences and Hospital and a private dental office in Mangalore. The sample size was chosen based on convenience. This sample size was amicable to the time allotted, resources available and ensured practicality and eliminate any ethical issues.

All the patients chosen for the present study required transalveolar extractions of horizontally impacted mandibular third molar in close proximity to IAN. Included in the study were 8 women and 12 men with a mean age of 25 years. An informed consent was obtained by all the participating subjects prior to the study.

\section{a) Criteria for Inclusion of Patients-}

- Patient willing to give informed consent.

- Patients of both sexes.

- Patients between 18 - 40 years of age.

- Patient indicated for transalveolar extraction of horizontally impacted mandibular third molar in close proximity to IAN.

\section{b) Criteria for Exclusion of Patients-}

- The presence of uncontrolled diabetes, immune disease or other contraindicating systemic conditions.

- Radiation therapy to the head and neck region in the 12 months before the proposed therapy.

- Chemotherapy in the 12 months period earlier the proposed therapy.

- Uncontrolled periodontal disease or patient's unwillingness to undergo needed periodontal therapy around remaining teeth.

- Presence of any local infection.

- A smoker.

- A psychological problem that in the opinion of the researcher would have rendered the delivery of comprehensive therapy untenable including depressed states and extreme nervousness or agitation that could preclude the patient from undergoing numerous lengthy treatment visits.

- An unwillingness to commit to a long-term follow-up program.

\section{Method of Data Collection}

The purpose of this study is to qualitatively evaluate the clinical procedural ease of the two-stage technique and infer its advantages if any. Therefore, a sample size of 20 was chosen as per convenience.

Twenty subjects satisfying the mentioned inclusion and exclusion criteria were included Since they constituted a homogeneous critical case study population, they were allocated into two groups of ten each arbitrarily based on no specific criteria to eliminate any bias.

One group of patients received the conventional mode of treatment for removal of impacted molar teeth (Control group) and the other, the two-stage treatment modality (Test group) respectively.

Control group $(n=10)$ : underwent conventional transalveolar extraction.

Test group ( $\mathrm{n}=10)$ : underwent two-stage technique.

Both the groups were post-operatively assessed for:

\section{IAN Injury13-14}

- Level A: Two-point discrimination test, Brush stroke direction.

- Level B: Contact detection/ light touch.

- Level C: Thermal testing, Sharp blunt discrimination, Pin prick test.

\section{Soft Tissue Healing One Week Post-Operatively}

- Pain.

- Swelling.

- Wound Dehiscence.

- Infection.

\section{Patient Compliance} Surgeon Satisfaction

All of the twenty patients were operated for removal of impacted teeth by the same operating surgeon. They were all assessed pre- and postoperatively for the neurosensory examination by another person blinded to the study. Soft tissue healing assessment, patient compliance and surgeon satisfaction were the operating surgeon's prerogative.

\section{Surgical Procedure: \\ Conventional Technique}

Under complete aseptic conditions, ample local anaesthesia was achieved by classical inferior alveolar nerve block with 2\% lignocaine with 1: 80,000 adrenaline. Ward's incision was placed and mucoperiosteal flap was raised. Lingual nerve was protected using a Howartz' periosteal elevator. Buccal bone ostectomy was done with carbide and diamond burs using Moore-Gillbe's buccal bone guttering technique under copious irrigation. Tooth was thereafter sectioned into crown and root, and further into mesial and distal root with a tapered fissure bur and the sections removed in order respectively. Socket was debrided with betadine and saline. After haemostasis, flap was replaced and secured into position with 3-0 silk. Patient was recalled after 1 week for suture removal and subsequently for designated followup.

\section{Two-Stage Technique}

Under complete aseptic conditions, ample local anaesthesia was achieved by classical inferior alveolar nerve block with 2\% lignocaine with 1: 80,000 adrenaline. Ostectomy was carried out with carbide and diamond burs to obtain access to the tooth. Then by use of a fissure bur, the anatomic crown was sectioned from the root and the sectioned crown part removed. Care was taken to avoid pulp exposure. At this point, the distance between the distal aspect of the second molar and the mesial aspect of the sectioned impacted tooth was measured and recorded to monitor the degree of migration of the right mandibular third molar. Before closing, a periapical radiograph of the area was obtained as a reference. Single interrupted resorbable No. 5-0 sutures (Vicryl; Ethicon, Somerville, NJ) were used to close the flap. A monthly checkup was scheduled for the next 3 months. At each visit, a new 
periapical radiograph was obtained. The mesial migration of the tooth and the clearance from the inferior alveolar canal was observed in the serial radiographs. Once the position of the tooth was satisfactory in terms of accessibility and at a safe position with clearance from IAN under local anaesthesia, a limited flap was raised without a vertical releasing incision and residual tooth was removed. The flap was then sutured back with 3-0 silk. A week later, the patient returned for suture removal.1,2

\section{Radiographic Analysis}

Orthopantomograph: To determine type of impaction and IAN proximity [Fig. 1].

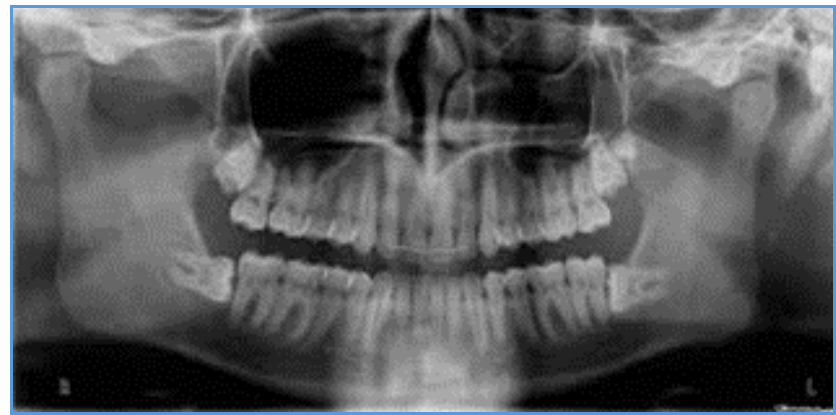

Figure 1. OPG showing Horizontally Impacted Left Mandibular Molar in "Intimate" relation to Mandibular Canal

\section{IOPA}

IAN relation to impacted tooth, mesial migration of residual tooth in two-stage technique [Fig. 2, 3, 4].

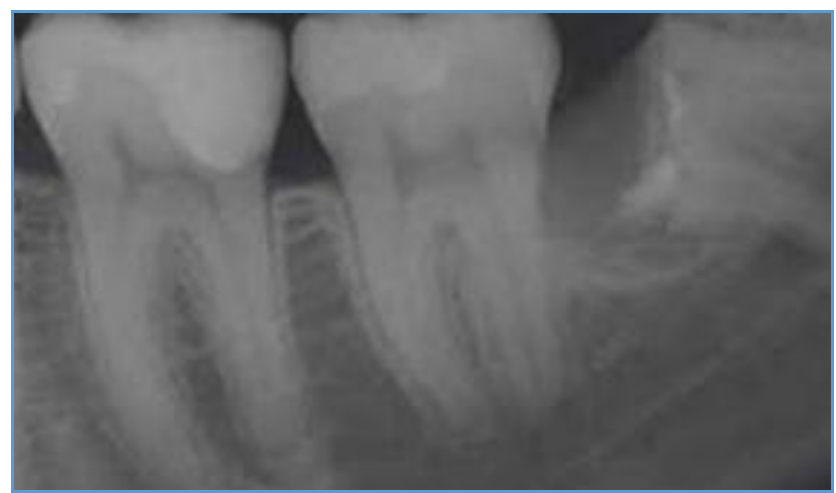

Figure 2. Odontectomy of Crown

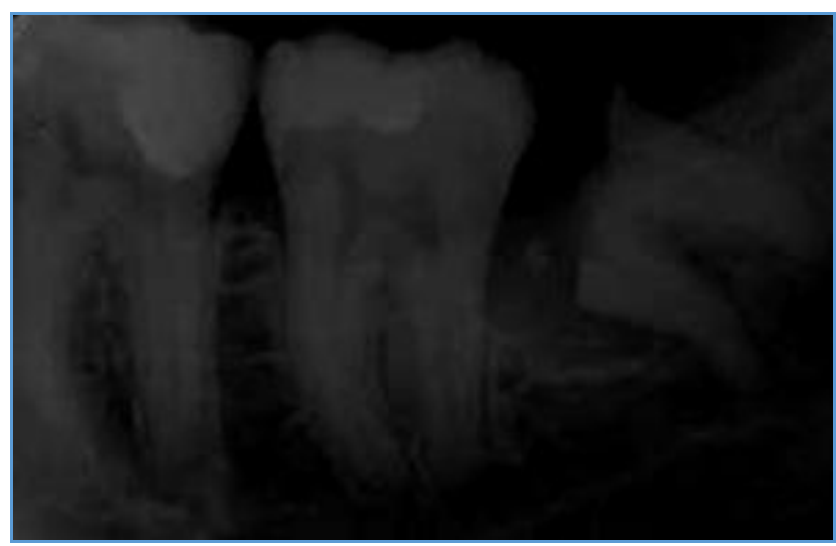

Figure 3. Mesial Migration of Tooth Residue in 2 Months

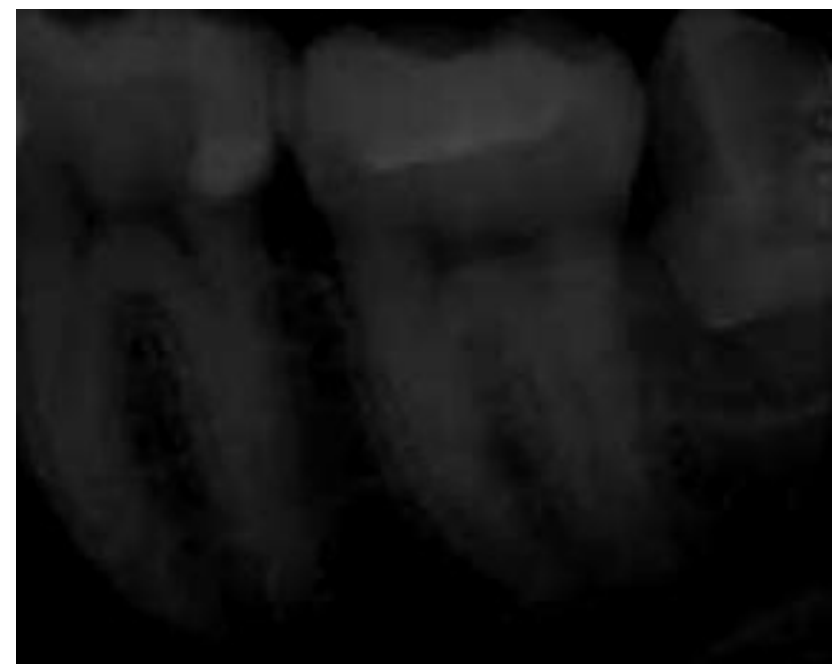

Figure 4. Mesial Migration of Tooth Residue after 3 Months

\section{Statistical Analysis}

The descriptive data was entered into MS Excel Sheet. Descriptive statistics were performed and frequency distribution was tabulated using statistical software SPSS (Statistical Package for Social Sciences) Version 20.1 (Chicago, USA, Inc.). Paired ' $\mathrm{t}$ ' test and two sample ' $\mathrm{t}$ ' test was used for the analysis of the tabulated data.

\section{Null Hypothesis}

There is no significant difference in the mean no. of patients having IAN injury between the two groups, i.e. $\mu_{1}=\mu_{2}$

\section{Alternate Hypothesis}

There is a significant difference in the mean no. of patients having IAN injury between the two groups, i.e. $\mu_{1} \neq \mu_{2}$

\section{Level of Significance- $\alpha=0.05$ \\ Decision Criterion}

The decision criterion is to reject the null hypothesis, if the pvalue is less than 0.05 . Otherwise, we accept the null hypothesis.

\section{RESULTS}

In order to evaluate nerve dysfunction, objective testing rather than to simply ask a patient to subjectively report neuropathic changes was used. Neurosensory disorders can be measured through various test levels as mentioned before.

The lip and chin were assessed by measuring the function of the IAN with light touch sensation. The contralateral halves of the lip and chin were taken as a control site. The area with impaired sensation was drawn on the skin. The patients with altered sensation returned 3 and 6 months postoperatively and their recovery pattern was noted. Neurosensory disturbances of the lip and chin were assessed by measuring the function of the IAN with light touch sensation.

Based on the neurosensory testing, temporary IANI occurred in 2 patients (10\%) of the control group and in 1 patient $(10 \%)$ in test group. Altered sensations lasting longer than 6 months was scored as permanent IANI (1 patient [5\%] of the control group). Levels $\mathrm{A}$ and $\mathrm{B}$ tests (two-point discrimination, brush-stroke direction and contact detection) were altered in 3 patients overall (15\%). In Level C tests (pinprick test and sharp blunt detection) showed that the nerve had recovered completely by 2 months [Table 1]. 


\begin{tabular}{|c|c|c|}
\hline & Control Group & Test Group \\
\hline No. of patients & 10 & 10 \\
\hline No. of IAN injury & 2 & 1 \\
\hline Soft tissue healing (total score) & 8 & 17 \\
\hline Patient compliance & Better & \\
\hline Surgeon's satisfaction & Better & \\
\hline Table 1. Comparison between Control and Test Groups \\
\hline
\end{tabular}

One patient (10\%) sustained permanent sensory loss. The lowest incidences were seen when the nerve was either "distant" or "close" and highest when the nerve was classed as "intimate." The difference between the "distant," "close" and "intimate" groups was statistically significant $(\mathrm{P}<0.01)$. There was no statistically significant difference between the two stage and the conventional group with respect to the occurrence of IAN injury [Table 2].

\begin{tabular}{|c|c|c|c|}
\hline \begin{tabular}{|c|} 
Position of the \\
Roots in Relation \\
the MC
\end{tabular} & Distant & Close & Intimate \\
\hline IAN injury incidence & - & $1 / 20(5 \%)$ & $2 / 20(10 \%)$ \\
\hline $\mathrm{P}$ value & 0.006 & $<0.0001$ & 0.32 \\
\hline \multicolumn{4}{|c|}{$\begin{array}{c}\text { Table 2. Paired 't' Test for IAN Injury in Relation to } \\
\text { Position of the Roots in Relation to the MC }\end{array}$} \\
\hline
\end{tabular}

MC: Mandibular Canal

The soft tissue healing were assessed qualitatively under the parameters of postoperative swelling, pain, infection and wound dehiscence a week following surgery and recorded in a questionnaire where the scales were absent (0), mild (1), moderate (2) and severe (3). Although, the results did not differ statistically, healing was rated better among the control cases in terms of tissue quality. This could be attributed to a second procedure involving incising the soft tissue in the same region [Table 3].

\begin{tabular}{|c|c|c|c|c|c|c|c|c|}
\hline Parameter Assessed & \multicolumn{2}{|c|}{ Absent (0) } & \multicolumn{2}{c|}{ Mild (1) } & \multicolumn{2}{c|}{ Moderate (2) } & Severe (3) \\
\hline & Control & Test & Control & Test & Control & Test & Control & Test \\
\hline Pain & 7 & 6 & 1 & 2 & - & & 2 & 2 \\
\hline Swelling & 9 & 7 & 1 & 1 & & & & \\
\hline Wound Dehiscence & 10 & 10 & & & & & & \\
\hline Infection & 10 & 9 & & & 1 & & \\
\hline \multicolumn{8}{|c|}{ Table 3. Soft Tissue Evaluation for Post-Operative Healing } \\
\hline
\end{tabular}

Patient compliance and surgeon satisfaction were at a higher level in the control cases, as this eliminated the second procedure and the lag time waiting for the tooth migration [Table 4].

\begin{tabular}{|c|c|c|c|c|c|c|c|}
\hline Patient Comfort & \multicolumn{1}{|c|}{ Intra-Operative } & Inter-Operative & \multicolumn{2}{c|}{ Post-Operative } & \multicolumn{2}{c|}{ Severe (3) } \\
\hline & Control & Test & Test & Control & Test & Control & Test \\
\hline Comfortable (1) & 8 & 9 & 5 & 7 & 9 & & \\
\hline Uncomfortable (2) & 2 & 1 & 5 & 3 & 1 & & \\
\hline \multicolumn{7}{|c|}{ Table 4. Patient Compliance } \\
\hline
\end{tabular}

\begin{tabular}{|c|c|}
\hline Proximity to the MC & Description \\
\hline Distant from the canal & There is a radiologic separation of $>1$ mm between the tips of the roots of the third molar and the MC \\
\hline Close to the canal & There is unchanged superimposition of the root apices over the MC \\
\hline Intimate to the canal & $\begin{array}{c}\text { The tips of the roots are considered to be intimate to the MC when } \geq 1 \\
\text { of the following 3 criteria are met: } 1 \text {. Diversion of the MC, 2. Darkening of the root, 3. Deflected root } \\
\text { apices. }\end{array}$ \\
\hline & \begin{tabular}{c} 
Table 5. Relationship of the Root's Apices to the Mandibular Canal (MC) \\
\hline
\end{tabular}
\end{tabular}




\begin{tabular}{|c|c|c|c|c|c|c|}
\hline \multirow[b]{2}{*}{ 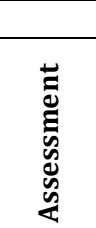 } & \multicolumn{2}{|c|}{ Level A tests } & \multirow{2}{*}{\begin{tabular}{|c|} 
Level B test \\
Assesses the \\
quickly adapting \\
large myelinated \\
(A-a) fibres
\end{tabular}} & \multicolumn{3}{|c|}{ Level C tests } \\
\hline & $\begin{array}{l}\text { This test is used to } \\
\text { determine the response of } \\
\text { the slowly adapting larger } \\
\text { myelinated fibres (A-a) }\end{array}$ & & & & $\begin{array}{c}\text { These tests assesses } \\
\text { the small myelinated } \\
\text { A-d and C fibres }\end{array}$ & \\
\hline $\begin{array}{l}\text { Test } \\
\text { Name }\end{array}$ & $\begin{array}{c}\text { Two-point discrimination } \\
\text { test }\end{array}$ & $\begin{array}{l}\text { Brush stroke } \\
\text { direction }\end{array}$ & $\begin{array}{c}\text { Contact detection/ } \\
\text { light touch }\end{array}$ & Thermal testing & $\begin{array}{c}\text { Sharp blunt } \\
\text { discrimination }\end{array}$ & Pin-prick test \\
\hline อ̃ & $\begin{array}{l}\text { Two pointed, but not sharp, } \\
\text { tips of a caliper touch the } \\
\text { skin simultaneously with } \\
\text { light pressure, while the } \\
\text { patient's eyes are closed. } \\
\text { The separation of the two } \\
\text { points are gradually } \\
\text { reduced from } 20 \text { mm at the } \\
\text { chin and } 10 \mathrm{~mm} \text { at the lips } \\
\text { to the moment where the } \\
\text { patient can feel one point } \\
\text { only. The minimum } \\
\text { separation at which two } \\
\text { points can be report is } \\
\text { recorded. }\end{array}$ & \begin{tabular}{|} 
The sensory \\
modalities for \\
these receptors are \\
vibration, touch \\
and flutter. Moving \\
stimuli is delivered \\
with a soft brush at \\
a fairly constant \\
velocity (2 - 3 \\
cm/s). Ten, two \\
interval forced \\
choice trials are \\
then delivered to \\
verify that the \\
direction of motion \\
is identified \\
correctly.
\end{tabular} & $\begin{array}{c}\text { The contact } \\
\text { detection } \\
\text { threshold, the } \\
\text { minimum force of } \\
\text { contact against the } \\
\text { skin that is felt is } \\
\text { measured with the } \\
\text { use of a } \\
\text { monofilament } \\
\text { mounted onto end } \\
\text { of a plastic handle. }\end{array}$ & \begin{tabular}{|c|} 
Perception of \\
warmth is \\
attributed to \\
the integrity of \\
A-d fibres and \\
cold to C fibres. \\
Two small glass \\
tubes \\
containing \\
water at $50^{\circ} \mathrm{C}$ \\
(warm) and \\
$15^{\circ} \mathrm{C}($ cold) \\
were used. The \\
report of each \\
stimulus, i.e. \\
cold versus hot \\
is recorded.
\end{tabular} & \begin{tabular}{|} 
This is done by \\
touching the test area \\
randomly with a sharp \\
or a blunt head of the \\
mechanical probe. A \\
rubber stopper is \\
centred at the end of \\
the dental probe, so \\
that when the tip is \\
pressed to the skin a \\
constant degree of skin \\
indentation is caused.
\end{tabular} & $\begin{array}{l}\text { Consisted of } \\
\text { repeating ten } \\
\text { touches with } \\
\text { an explorer } \\
\text { tip on the } \\
\text { chin skin and } \\
\text { performed } \\
\text { many times } \\
\text { whenever the } \\
\text { patient } \\
\text { accused of } \\
\text { the contact. }\end{array}$ \\
\hline
\end{tabular}

\section{DISCUSSION}

Lower third molar extraction is one of the most common procedures performed in oral and maxillofacial surgery units. The surgical procedure however is associated with various complications. Infections, fracture of the jaw, trismus and nerve injuries being the most common. The most worrisome of these complications however is the injury of the inferior alveolar nerve (IAN). Damage to the IAN during third molar extraction is a major concern for patients and clinicians. A wide range of the incidence of temporary and permanent neurologic disturbances of the IAN as a consequence of mandibular impacted third molar extraction has been reported in the literature. 1 The incidence of IAN injury reported in the literature ranges from $1.3 \%$ to $5.3 \% .^{2-5}$ It is most often a post-operative complication of disimpaction of horizontally impacted mandibular third molars. The risk of this complication depends mainly on the position of the impacted tooth in relation to the inferior alveolar canal

Pogrel and Thamby in 2000 estimated that every full-time practitioner will have one patient in their lifetime of work who will suffer from permanent nerve involvement as a result of IAN injury. ${ }^{6}$ The sensory branches of the trigeminal nerve encode information about facial expressions, speaking and chewing movements and stimuli that come into contact with the orofacial tissues. Whatever the cause, damage to the inferior alveolar nerve negatively affects the quality of facial sensibility as well as the patient's ability to translate patterns of altered nerve activity into functionally meaningful motor behaviours. ${ }^{6}$ Worldwide, the incidence of IAN is reported from $0.26 \%$ to $8.4 \%$.

\section{Several of the Risk Factors are listed below-}

1. Advanced age.

2. Difficulty of the operation.

3. Depth of tooth impaction.

4. Most important factor is the anatomic proximity of the third molar to the nerve canal. 7
Specifically, it is the lack of cortical integrity of the inferior alveolar canal in relation to the mandibular third molar roots. If there is no cortical bone between the IAN and the roots, there is $11.8 \%$ higher incidence of paraesthesia as compared to a case with an intact cortex of the IA canal. ${ }^{7}$

The incidence of inferior nerve injury is associated with the anatomical relationship between the $\operatorname{root}(\mathrm{s})$ of the third molar and the inferior alveolar nerve canal, and the rate of such nerve injury dramatically increases when the $\operatorname{root}(\mathrm{s})$ is close to the canal. ${ }^{10}$ The various relationship of impacted tooth root to the mandibular canal is explained in Table 5 . The relationship between the roots of mandibular teeth and the inferior alveolar nerve can often be assessed radiographically, particularly with a panoramic radiograph.1-3 Further, so it can be confirmed with an IOPA. Orthopantomography (panoramic radiography) is the radiologic investigation of choice before M3 surgery. The criteria for injury are identifiable on this radiograph, but like other conventional radiographs it is unable to give complete information in 3 dimensions. ${ }^{11}$

The issue of inferior alveolar nerve involvement during the removal of lower third molars is a clinical and medico-legal problem. Any technique that can reduce the possibility of this involvement is worthy of exploration. A modification of the surgical technique for lower third molar extraction is required to decrease the incidence of nerve injury, especially when the third molar is horizontally impacted and the root is close to the inferior alveolar nerve canal. Some authors advocated orthodontic-assisted extraction of the impacted mandibular third molars. -9 $^{-9}$ Others introduced partial odontectomy, the surgical removal of the anatomic crown leaving the roots in place. ${ }^{10-12}$ This case report describes a novel approach to the extraction of horizontally and mesially inclined impacted third molars with the root apexes in close contact with the IAN. Checchi and coworkers ${ }^{7,8}$ presented an interesting approach by using an orthodontic appliance to move the third molar away from the IAN. They reported on a case of deeply vertically impacted third molar in need of extraction. ${ }^{8}$ Five 
months after active orthodontic movement and 3 months after stabilisation they were able to extract the tooth without any neurologic consequences. However, the orthodontic device is applied in an area of the mouth that is very difficult to access, and it may cause compression and ulceration of the neighbouring tissues with a certain degree of discomfort.

Two stage approach is a novel surgical technique proposed by Landi et al This approach consisted of the surgical removal of the mesial portion of the anatomic crown to create adequate space for mesial migration of residual impacted tooth. It is aimed to provide adequate space distal to the second molar to allow spontaneous third molar eruption to displace the roots away from the neurovascular bundle. ${ }^{1}$ There is evidence in young adults that $26 \%$ to $35 \%$ of unerrupted mandibular third molars may change their position over time and reach the occlusal plane.10,11 This residual erupting activity of impacted third molars seems to be influenced by the pattern of impaction, because only 3\% of horizontally or mesially angulated impacted third molars with an angle of impaction greater than $35^{\circ}$ may spontaneously erupt compared with more than $30 \%$ of vertically positioned third molars. ${ }^{10}$ Therefore, it may be speculated that once space is provided mesial to the third molar, further migration of the tooth may be possible. This novel technique consists of a first surgical approach similar to the one adopted to extract impacted third molars. The amount of sectioning should take into account several factors: 1) Tooth position; 2) Degree of mesial shift desired to move the roots away from the nerve; and 3) Pulp chamber anatomy. Although, a generous amount of sectioning is desirable, every effort should be made at least during the first cut not to interfere with tooth vitality. In case of accidental pulp exposure, a pulpotomy may be done to minimise the risk of post-operative pain and discomfort.1,2

It may be speculated that this approach should be primarily used whenever the following features are present: 1) In case of horizontal or mesially inclined third molar impaction; 2) When radiographic evidence of the proximity of the third molar roots to the IAN is confirmed on a computed tomography scan; 3 ) When the crown of the third molar is in contact with the distal aspect of the second molar; 4) If an established pathologic process is detectable in the area of impaction (caries or deep periodontal defect) that indicates the need for third molar removal; 5) Preferably (but not exclusively) in young patients; and 6) Whenever orthodonticassisted extraction may be very complex to apply. This approach may also reduce chair time and cost compared with the orthodontic-assisted technique, while improving patient's comfort. Another advantage may be in case of tooth ankylosis. $^{2}$

Neurosensory disorders can be measured through various test levels [Table 6]. Neurosensory disturbances of the lip and chin were assessed by measuring the function of the IAN with light touch sensation.

\section{Nerve Injuries may affect}

1. Mechanoreception (touch pressure, positional sense);

2. Thermoreception (hot, cold);

3. Nociception (pain).

These sensations must be assessed to monitor whether a sensory disturbance is persistent or improving. Robinson et al in 1992 described a method of sensory testing and with this in mind a similar way of testing may be employed using everyday equipment in the general dental practice. ${ }^{15}$ Firstly, a subjective assessment involves asking the patient about the severity of their symptoms. Hillerup in 2008 has shown that there is an excellent correlation between NSD expressed in the patient's words and the objective findings by clinicians. ${ }^{16}$ An objective assessment is achieved by testing the injured side and comparing it to the non-injured side, which is used as a control. The room should be quiet. The patient should close their eyes and raise their finger to indicate they feel a stimulus. ${ }^{17}$

\section{An Objective Assessment Involves}

1. Light touch perception ${ }^{15}$ using a cotton pellet to lightly touch the affected area, one can map and measure an area within which no stimulus can be felt.

2. Pain (pin-prick) perception- Repeat as above using a dental probe and again map the area of anaesthesia or pinch the lower lip within a tissue forceps to ascertain if the stimulus is felt. $3,18,17$

3. Two-point discrimination threshold- Place the tweezers on the skin and enquire if one point or two points can be felt. Lips can distinguish two points $2-4 \mathrm{~mm}$ apart, whereas skin over the lower border of the mandible can distinguish only $8-10 \mathrm{~mm}$. The two-point discrimination is usually higher on the injured side. 18

4. Thermal assessment Test the affected area using a cotton pellet firstly dipped in cold water, then in hot water.

5. Directional sense- Brush the area with a brush or probe asking the patient in which direction the instrument is moving. ${ }^{18,15}$

6. Pointed dull discrimination- Alternate between the blunt end and the sharp end of a probe and ask the patient if the sensation is sharp or dull.

7. Location of touch- Ask the patient to point to the area that has just been touched by a probe. ${ }^{17}$

8. Photograph- By taking a photo of the mapped area, this may be used for comparison at the next visit. It is difficult to achieve reproducibility with photos, so reproducible markings should be placed.

Robinson et al stated that light touch stimuli, pain (pinprick) stimuli and two-point discrimination thresholds are adequate to detect evidence of early sensory recovery and that the latter two tests are the most likely to reveal a persistent sensory neuropathy. A patient's objective assessment may disimprove at a future visit and this may be a sign that a neuroma has formed, which is interfering with nerve conduction. ${ }^{18}$

A review of the literature indicates that nerve injuries occur following between 0.6 and 11 percent of third molar removals. ${ }^{19-21}$ Most patients recover fully without treatment and one study shows over 96 percent of the inferior alveolar nerve injuries and 87 percent of lingual nerve injuries recover spontaneously. ${ }^{22}$ The higher incidence of inferior alveolar nerve recovery is probably due to the fact that the nerve is retained within a bony canal and the damaged nerve endings. There appears to be general agreement that the majority of spontaneous recovery occurs within nine months, and that after two years there is very little likelihood of further spontaneous recovery. There are, however, well documented reports of occasional spontaneous recovery occurring several 
years after injury so that possibility cannot be dismissed At the current time, there are no modalities known for enhancing nerve regeneration.

\section{CONCLUSION}

Inferior alveolar nerve paraesthesia occurs widely from $0.35 \%$ to $8.4 \%$. Although, these figures are relatively low, they are still of great significance for both patients and clinicians. Mostly these injuries are unavoidable and hence all patients must be warned of the risks of mandibular third molar surgery including possible damage to the inferior alveolar nerve and informed consent must be obtained before the procedure.

Although, the incidence of IAN injury is found to be lower in the test group, it is not significantly different from that of the control group. The parameters of post-operative soft tissue healing, patient compliance and surgeon satisfaction all favour the control group. This could possibly be attributed to the disadvantages of the two-stage procedure, wherein a need for a second surgery, possibility of development of pathology due to retained tooth residue, post-operative tooth hypersensitivity induced by the odontectomy and the long duration for treatment completion.

Hence, with the findings of our study we would like to state that conventional technique is always more favoured technique bearing in mind that IAN injury has to be avoided. However, the two-stage technique can definitely be considered in cases of young patients in whom neurological deficits are unwarranted for. Ankylosed teeth and horizontally impacted mandibular third molars that have a high risk of IAN paraesthesia may also be dealt with in a two-stage fashion. This staged approach may be promising and further investigations may be warranted to evaluate its efficacy in a large sample of patients.

\section{REFERENCES}

[1] Landi L, Manicone PF, Piccinelli S, et al. Staged removal of horizontally impacted third molars to reduce risk of inferior alveolar nerve injury. J Oral Maxillofac Surg 2010;68(2):442-6.

[2] Kubota Y, Oka S, Yahara Y, et al. Two-stage method for the extraction of a horizontally impacted lower third molar. Oral Science International 2008;5(2):73-7.

[3] Libersa P, Savignat M, Tonnel A. Neurosensory disturbances of the inferior alveolar nerve: a retrospective study of complaints in a 10-year period. J Oral Maxillofac Surg 2007;65(8):1486-9.

[4] Bataineh AB. Sensory nerve impairment following mandibular third molar surgery. J Oral Maxillofac Surg 2001;59(9):1012-7.

[5] Kipp DP, Goldstein BH, Weiss WW. Dysesthesia after mandibular third molar surgery: a retrospective study and analysis of 1,377 surgical procedures. J Am Dent Assoc 1980;100(2):185-92.

[6] Goldberg MH, Nemarich AN, Marco WP. Complications after mandibular third molar surgery: a statistical analysis of 500 consecutive procedures in private practice. J Am Dent Assoc 1985;111(2):277-9.

[7] Smith AC, Barry SE, Chiong AY, et al. Inferior alveolar nerve damage following removal of mandibular third molar teeth: a prospective study using panoramic radiography. Aust Dent J 1997;42(3):149-52.
[8] Bui CH, Seldin EB, Dodson DB. Types, frequencies and risk factors for complications after third molar extraction. J Oral Maxillofac Surg 2003;61(12):137989.

[9] Checchi L, Bonetti GA, Pelliccioni GA. Removing of high-risk impacted mandibular third molars: a surgical orthodontic approach. J Am Dent Assoc 1996;127(7):1214-7.

[10] Bonetti GA, Bendandi M, Laino L, et al. Orthodontic extraction: riskless extraction of impacted lower third molars close to the mandibular canal. J Oral Maxillofac Surg 2007;65(12):2580-6.

[11] Hirsch A, Shteiman S, Boyan BD, et al. Use of orthodontic treatment as an aid to third molar extraction: a method for prevention of mandibular nerve injury and improved periodontal status. J Periodontol 2003;74(6):887-92.

[12] O'Riordan BC. Coronectomy (intentional partial odontectomy of lower third molars). Oral Surg Oral Med Oral Pathol Oral Radiol Endod 2004;98(3):274-80.

[13] Pogrel MA, Lee JS, Muff DF. Coronectomy: a technique to protect the inferior alveolar nerve. J Oral Maxillofac Surg 2004;62(12):1447-52.

[14] Renton T, Hankins M, Sproate C, et al A randomized controlled clinical trial to compare the incidence of injury to the inferior alveolar nerve as a result of coronectomy and removal of mandibular third molars. Br J Oral Maxillofac Surg 2005;43(1):7-12.

[15] Rolke R, Magerl W, Campbell KA, et al. Quantitative sensory testing: a comprehensive protocol for clinical trials. Eur J Pain 2006;10(1):77-88.

[16] Hillerup S, Jensen R. Nerve injury caused by mandibular block analgesia. Int J Oral Maxillofac Surg 2006;35(5):437-43.

[17] Walk D, Sehgal N, Moeller-Bertram T, et al. Quantitative sensory testing and mapping: a review of non-automated quantitative methods for examination of the patient with neuropathic pain. Clin J Pain 2009;25(7):632-40.

[18] Robinson PP. Observations on the recovery of sensation following inferior alveolar nerve injuries. $\mathrm{Br}$ J Oral Maxillofac Surg 1988;26(3):177-89.

[19] Howe GL, Poynton HG. Prevention of damage to the inferior dental nerve during the extraction of the mandibular third molars. Br Dent J 1960;109:353-63.

[20] Muhonen A, Ventä I, Ylipaavalniemi P. Factors predisposing to postoperative complications related to wisdom tooth surgery among university students. J Am Coll Health 1997;46(1):39-42.

[21] Rood JP. Lingual split technique. Damage to inferior alveolar and lingual nerves during removal of impacted mandibular third molars. Br Dent J 1983;154(2):4023.

[22] Rood JP, Shehab BA. The radiologic prediction of inferior alveolar nerve injury during third molar surgery. Br J Oral Maxillofac Surg 1990;28(1):20-5. 